\title{
A NADE nomogram to predict the probability of 6-month unfavorable outcome in Chinese patients with ischemic stroke
}

Chao Sun ${ }^{1,2+}$, Xiang Li ${ }^{1,2+}$, Baili Song ${ }^{1,2}$, Xiangliang Chen ${ }^{3}$, Linda Nyame ${ }^{1,2}$, Yukai Liư ${ }^{3}$, Dan Tang ${ }^{1}$, Mako Ibrahim ${ }^{1,2}$, Zheng Zhao ${ }^{2}$, Chao Liu' ${ }^{2}$, Miao Yan ${ }^{4}$, Xiding Pan², Jie Yang ${ }^{5}$, Junshan Zhou ${ }^{3^{*}}$ and Jianjun Zou ${ }^{2^{*}}$ (D)

\begin{abstract}
Background: Early prediction of unfavorable outcome after ischemic stroke is of great significance to the clinical and therapeutic management. A nomogram is a better visual tool than earlier models and prognostic scores to predict clinical outcomes, which incorporates different factors to develop a graphic continuous scoring system and calculates accurately the risk probability of poor outcome entirely based on individual characteristics. However, to date, no nomogram models have been found to predict the probability of 6-month poor outcome after ischemic stroke. We aimed to develop and validate a nomogram for individualized prediction of the probability of 6-month unfavorable outcome in Chinese patients with ischemic stroke.
\end{abstract}

Methods: Based on the retrospective stroke registry, a single-center study which included 499 patients from May, 2013 to May, 2018 was conducted in Nanjing First Hospital (China) for ischemic stroke within $12 \mathrm{~h}$ of symptoms onset. The main outcome measure was 6-month unfavorable outcome (mRS > 2). To generate the nomogram, $\mathrm{NIHSS}$ score on admission, Age, previous Diabetes mellitus and crEatinine (NADE) were integrated into the model. We assessed the discriminative performance by using the area under the curve (AUC) of receiver-operating characteristic (ROC) and calibration of risk prediction model by using the Hosmer-Lemeshow test.

Results: A visual NADE nomogram was constructed that NIHSS score on admission (OR: 1.190, 95\%Cl: 1.125-1.258), age (OR: 1.068, 95\%Cl: 1.045-1.090), previous diabetes mellitus (OR: 1.995, 95\%Cl: 1.236-3.221) and creatinine (OR: $1.010,95 \% \mathrm{Cl}: 1.002-1.018$ ) were found to be significant predictors of 6 -month unfavorable outcome after acute ischemic stroke in Chinese patients. The AUC-ROC of nomogram was 0.791. Calibration was good ( $p=0.4982$ for the Hosmer-Lemeshow test).

Conclusion: The NADE is the first nomogram developed and validated in Chinese ischemic stroke patients to provide an individual, visual and precise prediction of the risk probability of 6-month unfavorable outcome.

Keywords: Stroke, Cerebral ischemia, Unfavorable outcome, Prediction, Nomogram

\footnotetext{
*Correspondence: zoujianjun100@126.com; zhjsh333@126.com

${ }^{+}$Chao Sun and Xiang Li contributed equally to this work.

${ }^{2}$ Department of Clinical Pharmacology, Nanjing First Hospital, Nanjing

Medical University, Nanjing, Jiangsu, China

${ }^{3}$ Department of Neurology, Nanjing First Hospital, Nanjing Medical University,

Nanjing, China

Full list of author information is available at the end of the article
}

(c) The Author(s). 2019 Open Access This article is distributed under the terms of the Creative Commons Attribution 4.0 International License (http://creativecommons.org/licenses/by/4.0/), which permits unrestricted use, distribution, and reproduction in any medium, provided you give appropriate credit to the original author(s) and the source, provide a link to the Creative Commons license, and indicate if changes were made. The Creative Commons Public Domain Dedication waiver (http://creativecommons.org/publicdomain/zero/1.0/) applies to the data made available in this article, unless otherwise stated. 


\section{Background}

Globally, stroke is now the second most common cause of death and a major cause of disability $[1,2]$, and despite a reduction in mortality and hospitalization owing to improved acute management and implementation of preventive measures, the rate of stroke is on the rise owing to aging population. Therefore, early prediction of unfavorable outcome after ischemic stroke is of great significance to provide a reasonable approach to the clinical and therapeutic management and also help the patients and their families in understanding the challenging consequences of cerebral ischemia.

Nomogram is a visual statistical instrument that incorporates different data to develop a continuous scoring system which reflects the individual and precise risk probability. The accurate continuous probability power of the score emphasizes the potential of nomogram score as an important part of modern medical decision-making and a useful risk stratification tool that has been applied in routine clinical practice including cancer and surgery [3-6].

To our knowledge, there are only three researches on nomograms for individualized prediction of poor outcome of ischemic stroke in Caucasians (Italian) [7-9]. The START nomogram is developed and validated for individualized prediction of 3-month unfavorable outcome in intravenous thrombolysis-treated stroke patients [7]. Another nomogram model was established in 344 patients who started oral anticoagulants 1-7 days after atrial fibrillation-related stroke onset to predict the probability of 3-month poor outcome [8]. They found that NIHSS score which reflects the severity of stroke is the strongest predictor of 3-month unfavorable outcome [7-9]. However, to date, no nomogram model has been established to predict the probability of 6-month (longterm) unfavorable outcome after ischemic stroke in Chinese patients. Furthermore, the correlation of NHISS score with the outcomes varies with the time passed from the onset of cerebral ischemia [10]. It is unclear how National Institute of Health stroke scale (NHISS) score and other risk factors affect the 6-month (longterm) unfavorable functional outcome.

The objectives of this study were to develop and validate a nomogram model based on the integration of different degrees of stroke severity and other risk factors for individualized prediction of the probability of 6month (long-term) unfavorable functional outcome after ischemic stroke in Chinese patients, which can help direct individual treatment for ischemic stroke patients.

\section{Methods}

\section{Study design, participants, and procedures}

Based on the retrospective stroke registry, a singlecenter study was conducted in Nanjing First Hospital, Nanjing Medical University, China. The study protocol was approved by the hospital medical ethics committee in accordance with the Helsinki declaration. All of the consecutive patients were recorded in the stroke registry between May, 2013 and May, 2018 for ischemic stroke within $12 \mathrm{~h}$ of symptoms onset.

All patients managed with endovascular procedures were excluded from the study. Patients with signs of intracranial hemorrhage $(\mathrm{ICH})$ on baseline brain computed tomography (CT) scan, age $<18$ years, lack of 6month modified Rankin Scale (mRS) score and NIHSS score on admission unknown were excluded from the analysis. On admission, all clinical, demographic and laboratory characteristics were collected, including: age, sex, baseline NIHSS score, creatinine, platelet count, total cholesterol, fasting blood glucose, interval from onset to hospital within $4.5 \mathrm{~h}$, medical history such as diabetes mellitus, hypertension, valvular heart disease, cerebral hemorrhage and so on. The quality of laboratory data was validated throughout the study period by regular internal quality control procedures and participation to an External Quality Assessment scheme. Baseline NIHSS and 6-month mRS were assessed by trained physicians with telephone questionnaires or face-to-face interviews. Mortality, and any complications were recorded. The primary outcome was unfavorable functional outcome after ischemic stroke, as reflected by a mRS comprised between 3 and 6 (i.e., poor prognosis) at 6-month [11].

\section{Statistical analysis}

Baseline characteristics were summarized with descriptive statistics, continuous variables were reported as median value with interquartile range or means with standard deviation $[\mathrm{SD}]$. Categorical variables were instead expressed as number of events and percentage, dividing the number of events by the total number excluding missing and unknown cases. Differences between the two groups were explored using the Mann-Whitney U-test or Student $t$ test for continuous variables. Differences between proportions were assessed by Fisher's exact test or the $x^{2}$ test, when appropriate.

A NADE nomogram model was generated to predict the probability of 6-month unfavorable outcome. To develop a nomogram, the pre-established predictors and all variables with a probability value $<0.20$ in the univariate analysis were entered into a multivariate logistic regression model. A final model selection was carried out by a backward stepdown selection process with the Akaike information criterion. Regression coefficients and odds ratios (OR) with two-sided 95\% confidence intervals (95\% CIs) for each of the variable included in the model were finally calculated. Collinearity of combinations of variables that entered the multivariate logistic regression analysis was assessed by the Condition Index (<30 being considered non-significant) 
and Variation Inflation Factors (VIF, $<2$ being considered non-significant). The performance of the model was assessed by discrimination (the ability of a proposed model to separate patients with different outcomes) and calibration (the relative distance of predictions from actual outcome). The predictive accuracy of the nomogram model was assessed by calculation of the area under curve (AUC) of the receiver-operating characteristic (ROC). Calibration was carried out using a calibration plot, in which the predicted probabilities were plotted against the frequency of the observed unfavorable outcome. The prediction of a well-calibrated model should be mirrored by a $45^{\circ}$ diagonal line. Given that all predictive equations tend to be overfitting to the original sample, the model was internally validated using bootstrap resampling. The statistical analysis was carried out using SPSS version 22.0 (IBM Corporation, Armonk, NY, USA), Stata version 13.0 (Stata Corporation, College Station, TX, USA) statistical software and the statistical software package $\mathrm{R}$, version 3.3.3 (R Development Core Team, Auckland, New Zealand). All tests were two sided and $P<0.05$ was considered statistically significant.

\section{Results}

Based on the retrospective stroke registry, a single-center study from May, 2013 to May, 2018 was conducted in Nanjing First Hospital (China) for ischemic stroke within $12 \mathrm{~h}$ of symptoms onset. The final population consisted of 499 patients for acute ischemic stroke throughout the study period (median age 69 years; IQR $60-78$ years). Unfavorable outcome (mRS score 3-6) after 6-month from acute ischemic stroke was observed in 140 (28.1\%) patients, and within the follow-up period no patients died $(\mathrm{mRS}$ score $=6)$. The clinical, demographic and laboratory characteristics of the patients in the favorable outcome cohorts $(n=359)$ and unfavorable outcome $(n=140)$ cohorts are shown in Table 1 and Additional file 1: Table S1. In addition to age (65 versus 77; $p<0.0001$ ), NIHSS score on admission ( 2 versus $5 ; p<0.0001$ ), previous diabetes mellitus ( 89 versus $54 ; p=0.002)$ and creatinine (71 versus 78 ; $p=0.003)$, other factors such as gender, coronary artery disease, atrial fibrillation, previous stroke, previous antiplatelet, smoking, drinking, diastolic blood pressure (BP), international normalized ratios (INR), fasting blood glucose (FBG), triglyceride (TG), high density lipoprotein (HDL), and glycated hemoglobin (HbA1c) were also associated with unfavorable outcome in the univariate analysis. All variables with a probability value $<0.20$ in the univariate analysis and traditional stroke risk factors such as previous hypertension, systolic BP and Body Mass Index (BMI) were added into the multivariate regression model.

In multivariate analysis, NIHSS score on admission (OR: 1.190, 95\%CI: 1.125-1.258), age (OR: 1.068, 95\%CI: 1.045-1.090), previous diabetes mellitus (OR: 1.995, 95\%CI: $1.236-3.221$ ) and creatinine (OR: 1.010, 95\%CI: 1.002-1.018) were entered into a logistic regression model to construct the NADE nomogram for prediction

Table 1 Demographics and clinical characteristics according to 6-month outcome

\begin{tabular}{|c|c|c|c|}
\hline & Favorable outcome (mRS 0-2)) & Unfavorable outcome (mRS 3-6) & $P$ \\
\hline Patients, n(\%) & 359 & 140 & \\
\hline Sex, n(\%) & & & $0.021^{*}$ \\
\hline Male, n(\%) & $254(70.8)$ & $84(60.0)$ & \\
\hline Female, $n(\%)$ & $105(29.2)$ & $56(40.0)$ & \\
\hline \multicolumn{4}{|l|}{ Medical history, n (\%) } \\
\hline Hypertension & $249(69.4)$ & $105(75.0)$ & $0.212^{*}$ \\
\hline Diabetes mellitus & 89 (24.8) & $54(38.6)$ & $0.002^{*}$ \\
\hline Hyperlipidemia & $8(2.2)$ & $5(3.6)$ & 0.397 \\
\hline Coronary artery disease & $38(10.6)$ & $33(23.6)$ & $<0.0001^{*}$ \\
\hline Atrial fibrillation & $23(6.4)$ & $24(17.1)$ & $<0.0001^{*}$ \\
\hline Previous stroke & $68(18.9)$ & $42(30.0)$ & $0.007^{*}$ \\
\hline \multicolumn{4}{|l|}{ Baseline data } \\
\hline Age (years), median (IQR) & $65(58-74)$ & $77(70-83)$ & $<0.0001^{* a}$ \\
\hline NIHSS score, median (IQR) & $2(1-4)$ & $5(2-10)$ & $<0.0001^{* a}$ \\
\hline $\mathrm{BMI}, \mathrm{kg} / \mathrm{m}^{2}$, mean $(\mathrm{SD})$ & $24.79(3.25)$ & $24.51(3.76)$ & $0.407^{*}$ \\
\hline Systolic BP, mmHg, median (IQR) & $140(130-160)$ & $140(130-158)$ & $0.684^{* a}$ \\
\hline Creatinine, umol/L, median (IQR) & $71(60-84)$ & $78(63.25-95.25)$ & $0.003^{* a}$ \\
\hline
\end{tabular}

mRS modified Rankin Scale, NIHSS National Institute of Health stroke scale, BMI Body Mass Index

*included into the multiple logistic regression models $(P<0.2)$ and Additionally traditional stroke risk factors such as Hypertension, Systolic BP and BMI were added into the model.

${ }^{a}$ Calculated using Mann-Whitney $\mathrm{U}$ test. BMI Calculated using Student's tests 
of the probability of 6-month poor outcome in the cohort after the acute ischemic event (Table 2, Fig. 1). No significant statistical collinearity was observed for any of the four independent risk factors that entered the multivariate logistic regression analysis. The logistic regression model resulted: $\quad \log [\mathrm{p}(\mathrm{x}) / 1-\mathrm{p}(\mathrm{x})]=-7.388+(0.174 \times$ NIHSS score $)+(0.065 \times$ age $)+(0.691 \times$ previous diabetes mellitus) $+(0.010 \times$ creatinine $)$; where $\mathrm{p}(\mathrm{x})$ was the probability of 6-month unfavorable outcome.

The nomogram was developed by assigning a graphic initial score to each of the four independent prognostic factors with a point range from 0 to 100 , which was then summed to create a total score, finally converted into an individual risk of 6-month unfavorable outcome expressed in percentage, thus ranging from 0 to $100 \%$. It was predicted that the higher total score of the nomogram was associated with the higher likelihood of unfavorable outcome, while the lower total score was associated with the lower likelihood of adverse outcome. The AUC-ROC value of the NADE nomogram was 0.791 (95\% CI: 0.7460.836) in the cohort (Fig. 2). The age values and NIHSS scores on admission exhibited a good diagnostic accuracy for identifying patients with unfavorable outcome after 6 months, displaying an AUC of 0.734 (95\% CI 0.683-0.785; $p<0.0001$ ) and 0.713 (95\% CI 0.662-0.764; $p<0.0001$ ), respectively.

The total number of patients with a risk probability < $10 \%$ was $116 / 499$ (23.2\%), and only 9 of these had an unfavorable outcome (0.936 sensitivity, 0.298 specificity, 0.922 negative predictive value and 0.342 positive predictive value). The total number of patients with a risk probability < 40\% was 386/499 (77.4\%), 66 of whom had unfavorable outcome ( 0.529 sensitivity, 0.891 specificity, 0.829 negative predictive value and 0.655 positive predictive value). Finally, the total number of patients with a high-risk probability (i.e., $>80 \%$ ) was $25 / 499$ (5\%), the vast majority of whom $(21 / 25 ; 84 \%)$ had a poor prognosis (0.15 sensitivity, 0.989 specificity, 0.749 negative predictive value and 0.84 positive predictive value).

The model was internally validated using 1000 bootstrap samples to calculate the discrimination with accuracy, and the good predictive performance of the nomogram was confirmed, yielding a notable AUC of 0.791 (95\% CI 0.744-0.837; $p<0.0001$ ) (Fig. 2). The bias-corrected calibration plot for the nomogram model showed the

Table 2 significant predictors of 6-month unfavorable outcome after an acute ischemic stroke

\begin{tabular}{llllll}
\hline & OR & Error & Wald & $P$ & $95 \% \mathrm{Cl}$ \\
\hline Age & 1.068 & 0.012 & 6.07 & $<0.0001$ & $1.045-1.090$ \\
NIHSS on admission & 1.190 & 0.034 & 6.07 & $<0.0001$ & $1.125-1.258$ \\
Creatinine & 1.010 & 0.004 & 2.46 & 0.014 & $1.002-1.018$ \\
Previous Diabetes mellitus & 1.995 & 0.488 & 2.83 & 0.005 & $1.236-3.221$ \\
\hline
\end{tabular}

adequate agreement between predictors calculated with the NADE nomogram and actual unfavorable outcomes at the end of the follow-up period (Fig. 3). Calibration graphic revealed adequate fit of the model predicting the risk of poor prognosis at 6-month. The HosmerLemeshow goodness-of-fit test showed good calibration of the nomogram $(p=0.4982)$.

\section{Discussion}

Ischemic stroke is one of the leading causes of mortality and disability, and post-stroke disability has now become a public health care problem, so that the early and reliable identification of unfavorable outcome after ischemic stroke should be a valuable perspective on precise clinical and therapeutic management.

Some earlier models and prognostic scores identified that NIHSS score [12-16], age [12-14, 16, 17], creatinine $[18,19]$ and diabetes mellitus $[14,16,20]$, size infarct [8] were independent predictor of unfavorable outcome in stroke patients. However, these above models and scores were limited by the use of dichotomization / categorization of predictors, because the process of categorizing discrete/continuous variables (size infarct, age and NIHSS score) into 2 or 4 risk groups was often statistically inefficient and may decrease the predictive accuracy. Moreover, the disadvantage of dichotomization is that it does not make use of within-category information and lead to the loss of information.

Using continuous variables, we are the first to present a visual NADE nomogram which is a better tool for individualized prediction of the probability from 5 to $95 \%$ of 6 month poor outcome following ischemic stroke in Chinese patients, as shown in Fig. 1. The NADE nomogram, defined as graphical computation instrument composing four predictors that age, NIHSS score on admission, creatinine, and diabetes mellitus were observed to be independently associated with 6-month unfavorable outcome for individual ischemic stroke, and the effect remained significant with good discriminative performance even after adjusting other clinical, and demographic variables. That is, we found that NIHSS score on admission, age, previous diabetes mellitus and creatinine were significant and independent predictors of poor outcome. Moreover, patients age, NIHSS score on admission and creatinine are the non-categorical variables by order of decreasing multivariate predictive effect on the probability of 6-month poor outcome with good discriminative performance for individual stroke patients.

The NADE nomogram was a reliable model to evaluate the risk of 6-month mRS score 3-6 in stroke individuals, which showed a significant superiority for prediction of unfavorable outcome. Differently from prognostic scores, The NADE nomogram assigns a probability (from 5 to 95\%) of unfavorable outcome. For example, the NADE 


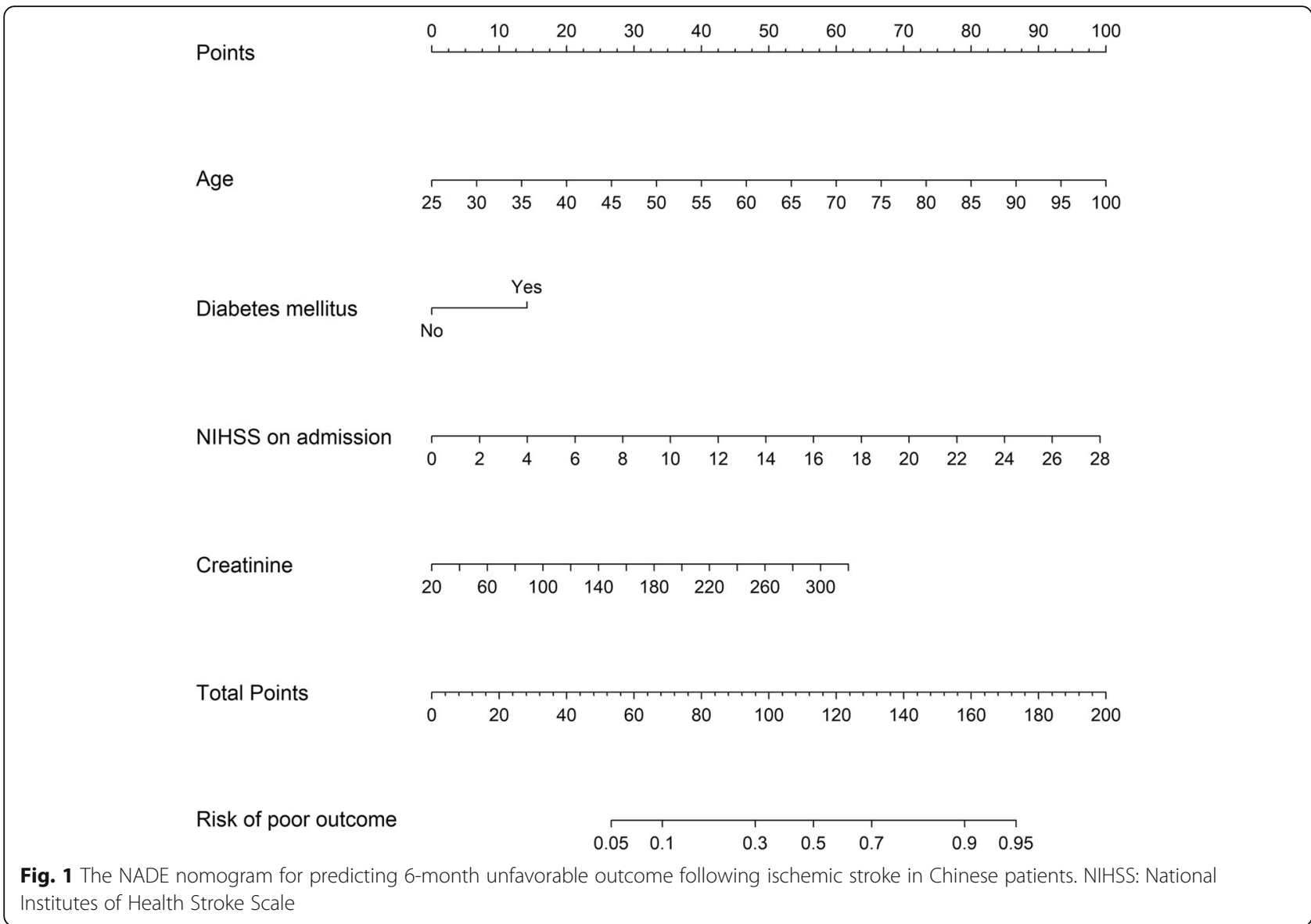

Fig. 1 The NADE nomogram for predicting 6-month unfavorable outcome following ischemic stroke in Chinese patients. NIHSS: National Institutes of Health Stroke Scale

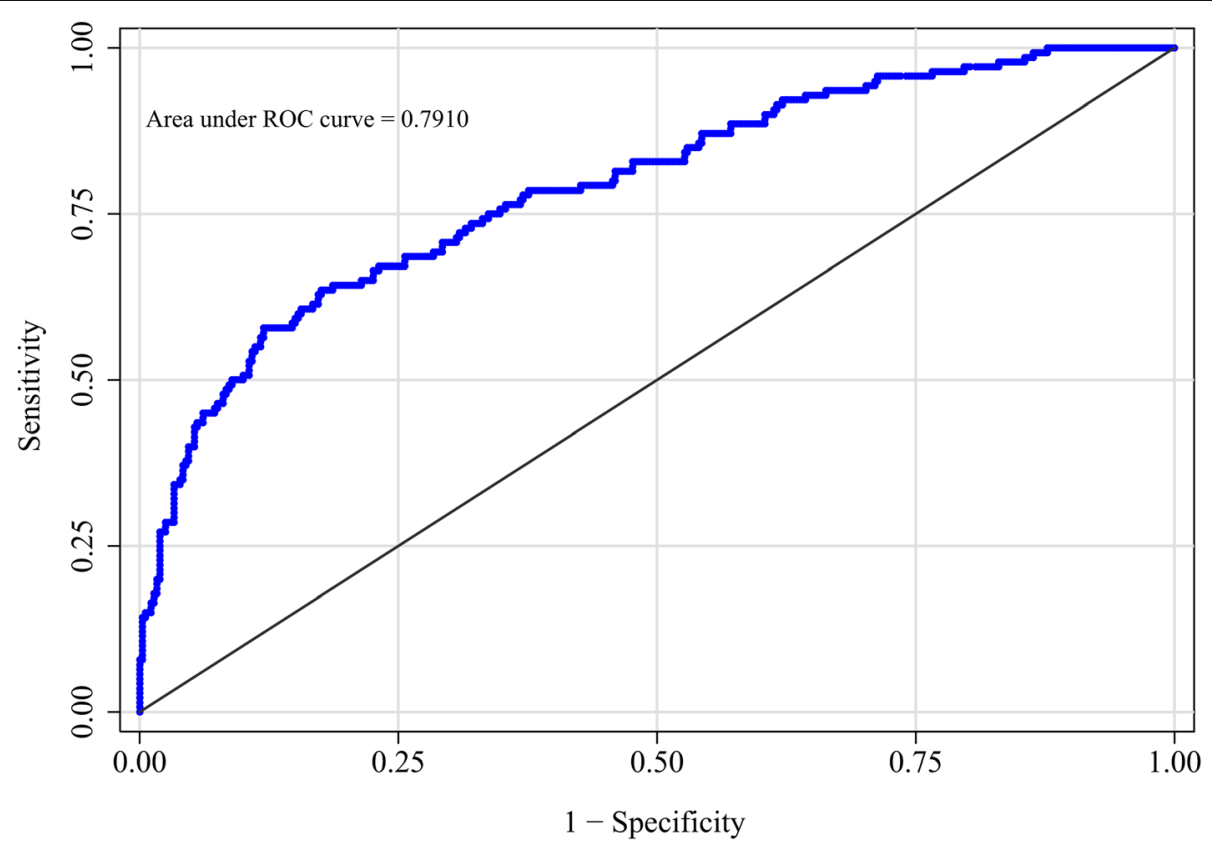

Fig. 2 Receiver operating characteristic (ROC) curve of the nomogram for predicting 6-month unfavorable outcome following ischemic stroke in Chinese patients 


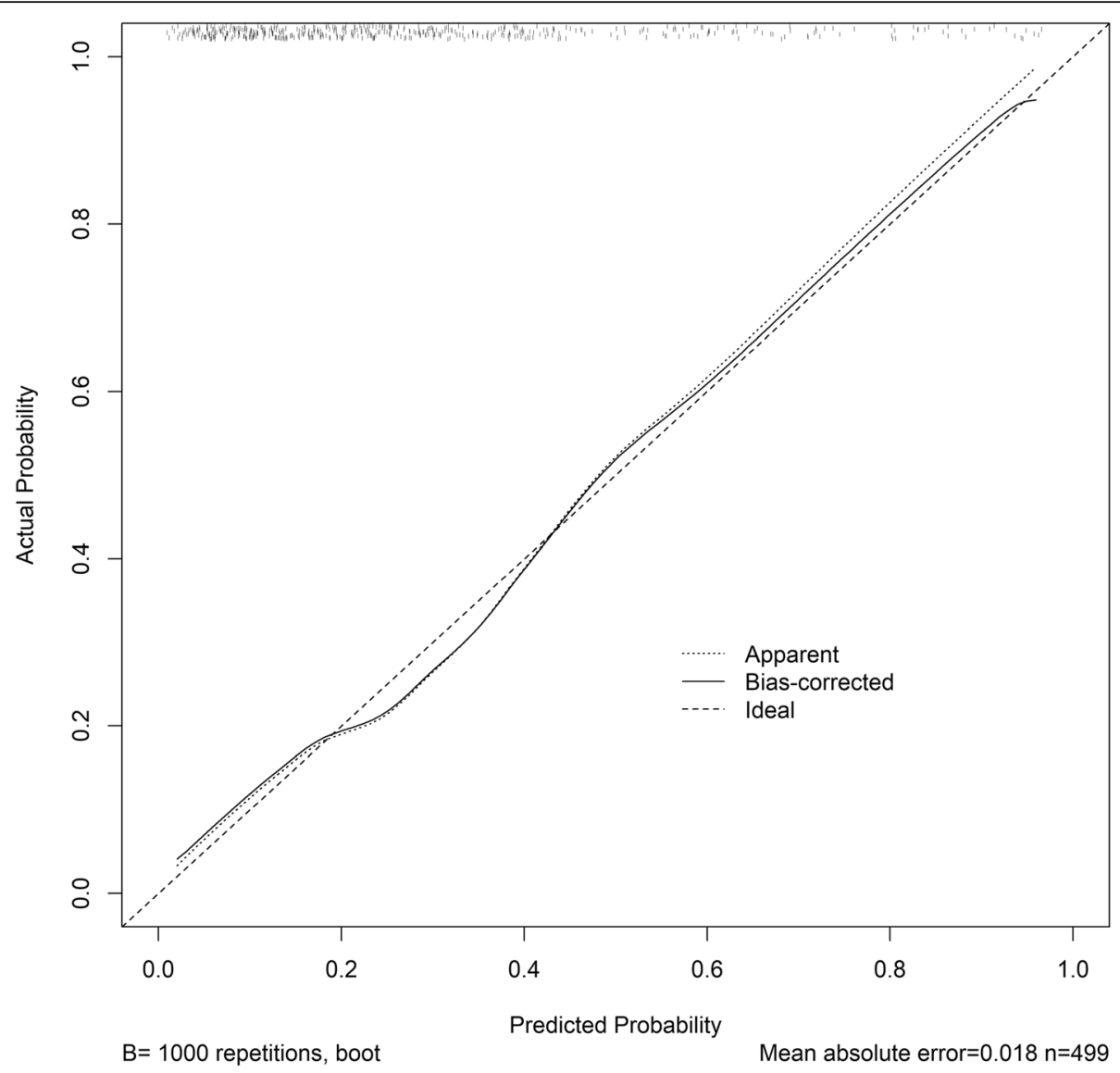

Fig. 3 The calibration plot for the nomogram for predicting 6-month unfavorable outcome following ischemic stroke in Chinese patients. Dashed line is reference line where an ideal nomogram would lie. Dotted line is the performance of nomogram, while the solid line corrects for any bias in nomogram

nomogram assigned $>95 \%$ probability of adverse consequence in an 80-year-old patient (74 points) stroke patient, with a history of diabetes mellitus (14 points), NHISS score of 10 (35 points) and creatinine level of 300 (58 points), with a total score of 181 points. On the other hand, $<10 \%$ probability of unfavorable outcome was nominated to a 40 years old ( 20 points), with no history of diabetes mellitus ( 0 points), NHISS score of 5 (18 points) and creatinine level of 100 (17 point), with a total score of 55 points. By converting the total score into a continuum of individual probability, the NADE nomogram can reclassify the risk of poor outcome more precisely. So the NADE nomogram could be better than previous prognostic scores and models based on outdated risk-grouping categorization for identifying different risk predictors in patients. Thus the NADE nomogram may provide more detailed information to facilitate the early identification of patients with very high probability of poor outcome and to discuss prognosis with patients and their families.

The NADE nomogram integrated patient age, NIHSS score, creatinine and diabetes mellitus for individualized prediction of 6-month unfavorable outcome following ischemic stroke in Chinese patients. Firstly, we found that age and NHISS on admission are the two strongest and independent predictors. For example, the NADE nomogram assigns a $45 \%$ probability of unfavorable outcome to a patient over 70 years old with modest creatinine and diabetes mellitus whose NIHSS score is 6 , respectively. Instead, 80 -year-old (74 points) and NHISS score of 10 (35 points) substantially influence the outcome in the patient because the probability of unfavorable outcome may remain higher than $75 \%$. Moreover, age contributed to a long-term mortality and poor outcome because old age is associated with some cardiovascular diseases such as coronary artery disease, high blood pressure and high cholesterol. In patients $>80$ years of age, the risk of poor outcome could be increased by the increased prevalence of cerebral small-vessel disease and comorbidities. Indeed, the NIHSS score indicates a more severe stroke, which can ultimately lead to a poorer outcome, whereas age means a less intense recovery. Secondly, the NADES nomogram also found that diabetes mellitus in ischemic stroke could predict worse outcome independently, because diabetes mellitus can 
affect the blood vessels and the nerves, which increases the chance of developing poor outcome. Finally, we observed that increased serum creatinine level was associated with unfavorable clinical outcome. Serum creatinine concentration is widely used as an index of renal function, and impaired renal function is associated with poor outcome after stroke [19].

Our study has some limitations. Firstly, it was an exploratory study in a single center and not a randomized controlled trial (RCT) designed to investigate the critical factors affecting 6-month poor outcome, and the clinical significance of this study may be attenuated by its design. Secondly, data of known neurobiological predictors such as infarct size [8] were not available in the present study, which may affect the development of the NADE nomogram model to predict the probability of 6-month poor outcome. So the neuroimaging predictors should also be integrated into our model in future studies, which may increase its discriminative performance of the nomogram model. Finally, an external validation in a completely different cohort of patients is warranted although the discriminative performance of the NADE model was good. Despite these limitations, as far as we know, the present study is the first attempt to construct and validate a nomogram to predict the probability of 6 month poor outcome in Chinese stroke patients.

\section{Conclusions}

The NADE nomogram may is a reliable tool with accurate continuous probability power to provide an individual and precise prediction of the risk probability of 6-month unfavorable outcome following ischemic stroke in Chinese patients.

\section{Supplementary information}

Supplementary information accompanies this paper at https://doi.org/10. 1186/s12883-019-1464-6.

Additional file 1: Table S1. Demographics and clinical characteristics according to 6-month outcome.

\section{Abbreviations \\ AUC: The area under the curve; BMI: Body Mass Index; BP: Blood pressure; Cl: Confidence interval; CT: Computed tomography; FBG: Fasting blood glucose; HbA1c: Glycated hemoglobin; HDL: High density lipoprotein; ICH: Intracranial hemorrhage; IQR: International normalized ratios; mRS: Modified Rankin Scale; NADE: NIHSS score on admission, Age, previous Diabetes mellitus and crEatinine; NIHSS: National Institute of Health stroke scale; OR: Odds ratios; RCT: Randomized controlled trial; ROC: Receiver- operating characteristic; SD: Standard deviation; TG: Triglyceride; VIF: Variation Inflation Factors}

\section{Acknowledgements}

We would like to thank all patients, researchers, and institutions for their collaboration and contribution to this study.

\section{Authors' contributions}

CS and XL contributed equally to this work. JSZ and JJZ concept, designed, and supervised the study. YKL, XLC, ZZ, and CL acquired the data. CS, XL, BLS and JJZ analyzed and interpreted the data, provided statistical analysis, had full access to all of the data in the study, and are responsible for the integrity of the data and the accuracy of the data analysis. LN, MI, DT, and CS drafted the manuscript, JJZ, JY, XDP, and MY critically revised the manuscript for important intellectual content. All authors read and approved the final manuscript.

\section{Funding}

The design of this study; the collection, analysis, and interpretation of data; and language editing were financially supported by National Natural Science Foundation of China grant 81673511, Jiangsu Key Research and Development Plan grant BE2017613, Jiangsu Six Talent Peaks Project grant WSN-151, and Nanjing Medical Science and Technique Development Foundation grant QRX17020 and ZKX15027.

\section{Availability of data and materials}

The data sets in this study are available from the corresponding author on reasonable request.

\section{Ethics approval and consent to participate}

The study protocols were approved by the Ethics Committees of Nanjing First Hospital in accord with the Helsinki declaration and internal protocol. All patients have given their written informed consent.

\section{Consent for publication}

Not applicable.

\section{Competing interests}

The authors declare that they have no competing interests.

\section{Author details}

${ }^{1}$ School of Basic Medicine and Clinical Pharmacy, China Pharmaceutical University, Nanjing, Jiangsu, China. ${ }^{2}$ Department of Clinical Pharmacology, Nanjing First Hospital, Nanjing Medical University, Nanjing, Jiangsu, China. ${ }^{3}$ Department of Neurology, Nanjing First Hospital, Nanjing Medical University, Nanjing, China. ${ }^{4}$ Department of Pharmacy, the Second Xiangya Hospital, Central South University, Changsha, China. ${ }^{5}$ Department of Neurology, the First Affiliated Hospital of Chengdu Medical College, Chengdu, China.

Received: 5 May 2019 Accepted: 12 September 2019 Received: 5 May 2019 Accepted: 12 Septem

\section{References}

1. Pruvost-Robieux E, Calvet D, Ben Hassen W, et al. Design and methodology of a pilot randomized controlled trial of Transcranial direct current stimulation in acute middle cerebral artery stroke (STICA). Front Neurol. 2018;9:816.

2. Stewart C, Subbarayan S, Paton P, et al. Non-pharmacological interventions for the improvement of post-stroke activities of daily living and disability amongst older stroke survivors: a systematic review. PLoS One. 2018;13(10): e0204774

3. Chang $B$, He W, Ouyang $H$, et al. A prognostic nomogram incorporating depth of tumor invasion to predict long-term overall survival for tongue squamous cell carcinoma with R0 resection. J Cancer. 2018;9(12):2107-15.

4. Ashfaq A, Kidwell JT, McGhan LJ, et al. Validation of a gastric cancer nomogram using a cancer registry. J Surg Oncol. 2015;112(4):377-80.

5. Zhou $C$, Shi $Y$, Chai $P$, et al. Contemporary update of overall prognosis and nomogram to predict individualized survival for Chinese patients with eyelid sebaceous carcinoma. EBioMedicine. 2018;36:221-8.

6. Moulla Y, Lyros O, Adolf D, Kaiser T. Dietrich a a nomogram based on clinical factors to predict the serum myoglobin levels following bariatric surgery. Obes Surg. 2018;28(6):1697-703.

7. Cappellari M, Turcato G, Forlivesi S, et al. The START nomogram for individualized prediction of the probability of unfavorable outcome after intravenous thrombolysis for stroke. Int J Stroke. 2018;13(7):700-6.

8. Cappellari M, Turcato G, Forlivesi $\mathrm{S}$, et al. Introduction of direct oral anticoagulant within 7 days of stroke onset: a nomogram to predict the probability of 3-month modified Rankin scale score $>2$. J Thromb Thrombolysis. 2018;46(3):292-8

9. Turcato G, Cervellin G, Cappellari M, et al. Early function decline after ischemic stroke can be predicted by a nomogram based on age, use of 
thrombolysis, RDW and NIHSS score at admission. J Thromb Thrombolysis. 2017;43(3):394-400.

10. Saver JL, Altman H. Relationship between neurologic deficit severity and final functional outcome shifts and strengthens during first hours after onset. Stroke. 2012;43(6):1537-41.

11. Cooray C, Mazya M, Bottai M, et al. External validation of the ASTRAL and DRAGON scores for prediction of functional outcome in stroke. Stroke. 2016; 47(6):1493-9.

12. Emberson J, Lees KR, Lyden P, et al. Effect of treatment delay, age, and stroke severity on the effects of intravenous thrombolysis with alteplase for acute ischaemic stroke: a meta-analysis of individual patient data from randomised trials. Lancet. 2014;384(9958):1929-35.

13. Ntaios G, Faouzi M, Ferrari J, et al. An integer-based score to predict functional outcome in acute ischemic stroke: the ASTRAL score. Neurology. 2012;78(24):1916-22.

14. Strbian D, Meretoja A, Ahlhelm FJ, et al. Predicting outcome of IV thrombolysis-treated ischemic stroke patients: the DRAGON score. Neurology. 2012;78(6):427-32.

15. Weimar C, Konig IR, Kraywinkel K, Ziegler A, Diener HC. Age and National Institutes of Health stroke scale score within 6 hours after onset are accurate predictors of outcome after cerebral ischemia: development and external validation of prognostic models. Stroke. 2004;35(1):158-62.

16. Vora NA, Shook SJ, Schumacher HC, et al. A 5-item scale to predict stroke outcome after cortical middle cerebral artery territory infarction: validation from results of the diffusion and perfusion imaging evaluation for understanding stroke evolution (DEFUSE) study. Stroke. 2011:42(3):645-9.

17. Luedi R, Hsieh K, Slezak A, et al. Age dependency of safety and outcome of endovascular therapy for acute stroke. J Neurol. 2014;261(8):1622-7.

18. MacWalter RS, Wong SY, Wong KY, et al. Does renal dysfunction predict mortality after acute stroke? A 7-year follow-up study. Stroke. 2002;33(6): 1630-5.

19. Yahalom G, Schwartz R, Schwammenthal Y, et al. Chronic kidney disease and clinical outcome in patients with acute stroke. Stroke. 2009: 40(4):1296-303

20. Desilles JP, Meseguer E, Labreuche J, et al. Diabetes mellitus, admission glucose, and outcomes after stroke thrombolysis: a registry and systematic review. Stroke. 2013;44(7):1915-23.

\section{Publisher's Note}

Springer Nature remains neutral with regard to jurisdictional claims in published maps and institutional affiliations.

Ready to submit your research? Choose BMC and benefit from:

- fast, convenient online submission

- thorough peer review by experienced researchers in your field

- rapid publication on acceptance

- support for research data, including large and complex data types

- gold Open Access which fosters wider collaboration and increased citations

- maximum visibility for your research: over $100 \mathrm{M}$ website views per year

At BMC, research is always in progress.

Learn more biomedcentral.com/submissions 